\title{
A Background Noise Reduction Technique using Adaptive Noise Cancellation for Microphone Arrays
}

\author{
Taylor B. Spalt ${ }^{1}$, Christopher R. Fuller ${ }^{2}$ \\ Vibration and Acoustics Laboratories, Virginia Tech, Blacksburg, Virginia 24061 \\ Thomas F. Brooks ${ }^{3}$, William M. Humphreys, $\mathrm{Jr}^{4}$ \\ NASA Langley Research Center, Hampton, Virginia 23681
}

\begin{abstract}
Background noise in wind tunnel environments poses a challenge to acoustic measurements due to possible low or negative Signal to Noise Ratios (SNRs) present in the testing environment. This paper overviews the application of time domain Adaptive Noise Cancellation (ANC) to microphone array signals with an intended application of background noise reduction in wind tunnels. An experiment was conducted to simulate background noise from a wind tunnel circuit measured by an out-of-flow microphone array in the tunnel test section. A reference microphone was used to acquire a background noise signal which interfered with the desired primary noise source signal at the array. The technique's efficacy was investigated using frequency spectra from the array microphones, array beamforming of the point source region, and subsequent deconvolution using the Deconvolution Approach for the Mapping of Acoustic Sources (DAMAS) algorithm. Comparisons were made with the conventional techniques for improving SNR of spectral and Cross-Spectral Matrix subtraction. The method was seen to recover the primary signal level in SNRs as low as $-29 \mathrm{~dB}$ and outperform the conventional methods. A second processing approach using the center array microphone as the noise reference was investigated for more general applicability of the ANC technique. It outperformed the conventional methods at the $-29 \mathrm{~dB}$ SNR but yielded less accurate results when coherence over the array dropped. This approach could possibly improve conventional testing methodology but must be investigated further under more realistic testing conditions.
\end{abstract}

\section{Nomenclature}

$c_{n} \quad=$ Finite Impulse Response filter coefficients at step $n$

$\hat{e}=$ steering vector for array to focus location

$e_{m} \quad=$ component of $\hat{e}$ for microphone $m$

$f \quad=$ frequency

$\Delta f \quad=$ frequency bandwidth resolution of spectra

$G_{m m}, \quad=$ cross-spectrum between $p_{m}$ and $p_{m}$

$\hat{G} \quad=$ matrix $(\mathrm{CSM})$ of cross-spectral elements $G_{m m}$,

$K=$ counting number of CSM averages

$m=$ microphone identity number in array

$m^{\prime} \quad=$ same as $m$, but independently varied

$M \quad=$ total number of array microphones

$n_{0} \quad=$ background noise present in primary ANC input

$n_{1}=$ background noise present in reference $\mathrm{ANC}$ input

$N \quad=$ total number of FIR filter weights

$p_{m} \quad=$ pressure time records from microphone $m$

$P_{m} \quad=$ Fourier Transform of $p_{m}$

$r_{c}=$ distance $r_{m}$ for $m$ being the center array microphone

$r_{m} \quad=$ coordinate distance to $m$

$s \quad=$ signal from primary sound source

\footnotetext{
${ }^{1}$ Student, AIAA Student Member

${ }^{2}$ NIA Samuel P. Langley Professor, AIAA Associate Fellow

${ }^{3}$ Senior Research Scientist, AIAA Fellow

${ }^{4}$ Senior Research Scientist, AIAA Senior Member
} 


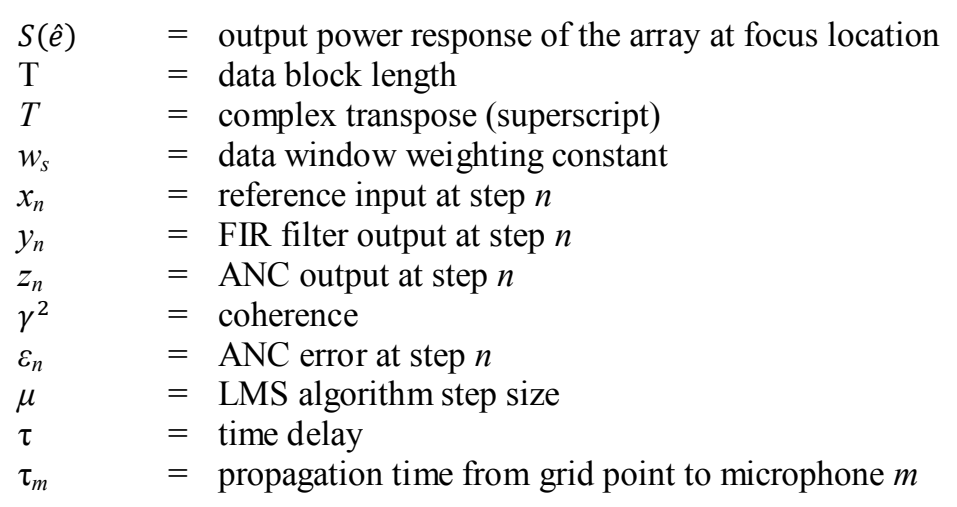

\section{Introduction}

NORMALLY designed wind tunnels are not optimal for acoustics testing because they are designed with only 1 aerodynamic considerations in mind. The acoustic measurements of components placed for testing in the tunnels are often masked by background noise generated by the wind tunnel flow drive or the flow itself, resulting in low or negative SNRs. This background noise limits the accurate measurement, identification, and quantification of noise sources under study. Thus, reduction of background noise to improve the SNR in testing environments is of interest.

In wind tunnels, techniques have been investigated that remove noise contamination from source noise measurements [1]. One technique used the assumption that if the background noise at two different transducers was uncorrelated yet statistically equal, the frequency spectrum due to only the model source could be recovered from the difference between the two time-series. Improvements were made on the technique over the next two decades [25]. In 1989, a technique for capturing model noise from turbulent boundary layer wall-pressure fluctuations was investigated [6]. The authors postulated that acoustic disturbances propagated down the wind tunnel acting as a waveguide. Turbulent pressure fluctuations due to local velocity disturbances were found to be uncorrelated to the acoustic disturbances at any streamwise location. Two microphones at the same streamwise location yet separated spanwise were used. One signal was delayed by a time constant $\tau$ then subtracted from the other signal to form a new time-series. The true spectrum due to the model source was obtained from this time-series at frequencies $1 / \tau$ and higher harmonics. It was concluded that some of the model source signal was cancelled along with the contaminating noise if the microphone spacing was insufficient. This approach was extended beyond the aforementioned frequency-domain studies to time-domain noise cancellation in 1996 [7]. The authors used an optimizing Finite Impulse Response (FIR) filter to estimate the correlated noise between a primary and reference signal then subtracted it from the reference signal. The turbulent pressure components in each signal were taken as uncorrelated as long as the transducers were separated by a sufficient distance [6]. Therefore, the correlated parts belonged to the common noise between the signals. This was then subtracted from the reference signal leaving just the source noise time-series with magnitude and phase intact. At low frequencies part of the source signal was cancelled along with the background noise if insufficient transducer spacing was used. It was shown that this undesired source signal cancellation [7] was an order of magnitude smaller than that removed using the aforementioned methods [1-6].

Early efforts in the study of time-domain Adaptive Noise Cancellation (ANC) were made in the 1960s [8,9] and the technique was formalized in 1975 [10]. The method used a minimum of two channels: a reference channel intended to receive only undesired background noise and a primary channel containing the signal plus background noise. The reference time-series is fed into a filter which is adapted to produce a best estimate of the noise present in the primary time-series and then subtracted from the primary signal leaving a "cleaned" result. Since its publication the technique has been widely employed primarily for applications of noise reduction for improved speech recognition. Note that Adaptive Noise Cancellation is an electronic, in-wire signal cancellation technique, as opposed to Active Noise Cancellation which involves actual pressure fluctuation cancellation.

An early, simplified experiment employing ANC reported at least $20 \mathrm{~dB}$ noise cancellation with minimum distortion [11]. Background noise in a fighter jet cockpit presented an important application of the technique that led to the identification of the major challenges for successful implementation. A reference microphone attached to the outside of the helmet was used to capture the ambient noise present in order to cancel it from the pilot's microphone. An ideal simulation [12] of the cockpit environment reported an $11 \mathrm{~dB}$ SNR improvement. An important consideration discussed was acquisition of the desired signal by the reference channel, which would result in 
cancellation of the desired signal at the output. This was circumvented by adapting the filter used for cancellation only during periods of background noise. A more realistic evaluation [13] of the cockpit environment was performed by simulating a diffuse background noise field and it was concluded that good cancellation was not possible at frequencies above $2 \mathrm{kHz}$. The study identified coherence between the reference and primary channels as the most important factor in successful cancellation. Increasing the distance between the transducers was cited as the cause for low coherence above $2 \mathrm{kHz}$. This was an effect of a diffuse sound field [14].

Experimental implementation of ANC appears to have conflicting requirements: good coherence is necessary for noise cancellation, necessitating the reference transducer be located either close to the noise source(s) (if localized) or close to the primary transducer(s) (if a diffuse noise field present). And if the noise source is not localizable, locating the reference transducer close to the primary may result in cancellation of the desired signal.

In microphone array applications, background noise has been removed using a spectral subtraction technique since 1974 [15]. The method relies on a separate acquisition of the background noise in the testing environment from that when the noise source under study is present. Upon converting signals to the frequency domain, this "background only" acquisition is subtracted (on a pressure-squared basis) from that of the primary acquisition (signal plus noise). With the introduction of beamforming using microphone arrays, this same principle has been applied to Cross-Spectral Matrices (CSMs) since the late 1990s [16-20]. Both techniques rely on the cancellation of the noise in its statistical sense, since the background noise present will not be identical during two acquisitions separated in time. The CSM subtraction technique allows further noise reduction beyond what spectral subtraction can achieve on a per channel basis. This is because uncorrelated noise at distinct array microphones is already reduced by the cross-correlation performed when forming the CSM [21]. These techniques are compared herein to ANC in an experiment intended to provide simulated wind tunnel background noise being measured by an out-offlow array in a wind tunnel test section.

\section{Theory}

Adaptive Noise Cancellation. Noise cancellation in the time domain utilizes a reference input, ideally containing just noise, which is passed through an adaptive filter and later subtracted from a primary input containing both the desired signal and correlated components of the noise present in the reference input. The minimized output becomes the primary signal with the noise attenuated or cancelled altogether. The correlation between the noise present in the reference channel and the primary input is important: the higher it is, the better the cancellation [13]. A diagram of the concept is pictured in Fig. 1.

In Fig. 1, a primary sound source $(s)$ that contains uncorrelated noise $\left(n_{0}\right)$ is transmitted to the upper left channel. The bottom left channel receives noise $\left(n_{l}\right)$ correlated with $n_{0}$ in some unknown manner. It is fed into the adaptive filter with the goal of replicating $n_{0}$. The output of the adaptive filter is subtracted from the primary input $\left(s+n_{0}\right)$ to produce $z$, which also serves as the error signal to the filter.

The interior of the dashed box of Fig. 1 is given in Fig. 2. Success of the adaptation is dependent on the causality of the system, i.e. the noise reference must be present in the system before the input. From Fig. 2, the reference signal $\left(x_{n}\right)$ is fed to the FIR filter at the start of the processing. The initial filter coefficients $\left(c_{n}\right)$ and number of weights are provided by the user. The output of the filter

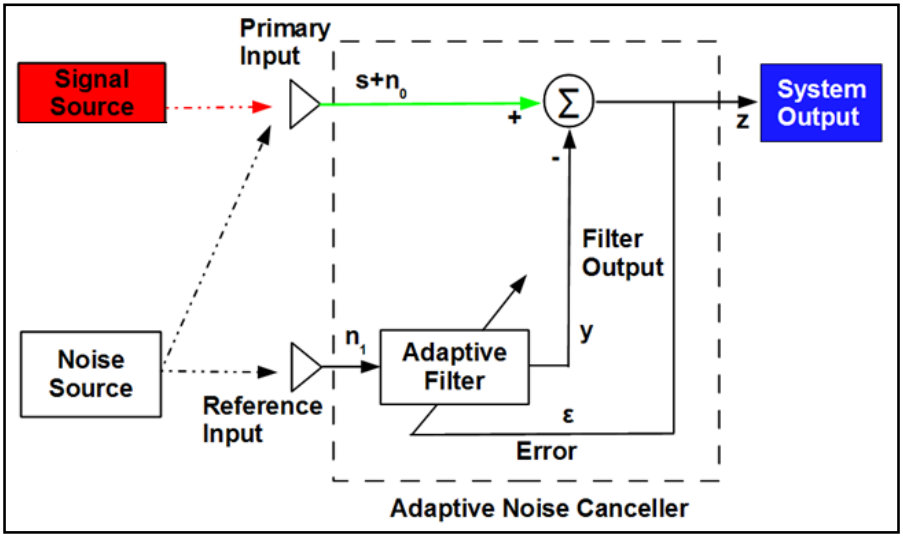

Figure 1. Adaptive noise canceller concept [10].

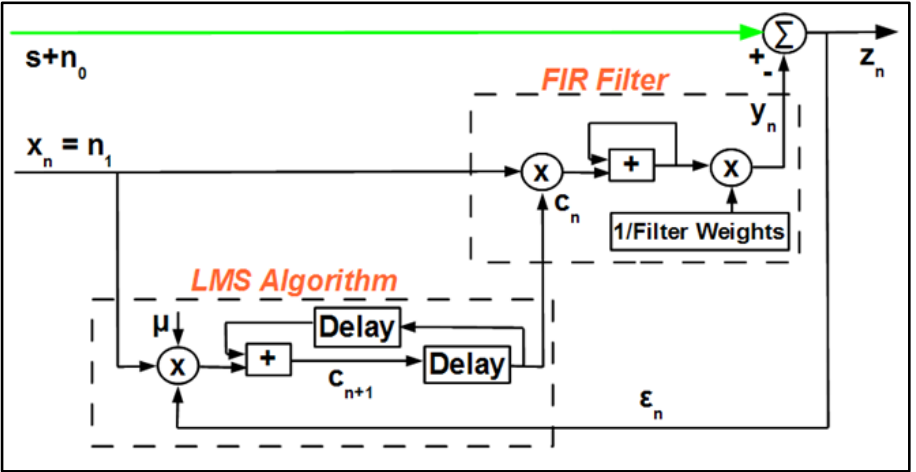

Figure 2. Adaptive noise canceller concept with LMS algorithm and FIR filter block diagrams included. 
$\left(y_{n}\right)$ is subtracted from the primary input signal $\left(s+n_{0}\right.$, an array microphone) which contains a correlated version of the noise present in the reference signal from an earlier time. The error $\left(\varepsilon_{n}\right)$ is then fed into the Least Mean Squares (LMS) algorithm [10] and multiplied with the reference input $\left(x_{n}\right)$ and the step size $(\mu)$ to produce the next set of filter coefficients $\left(c_{n+1}\right)$. The order of the FIR filter is the number of weights or "taps" used. FIR filters are always stable and have linear phase response given that the coefficients are symmetrical.

The difference equation defining the output of the filter is

$$
y_{n}=c_{0} x_{n}+c_{1} x_{n-1}+\cdots+c_{N} x_{n-N}
$$

where $N$ is the number of filter weights specified. As the noise to be cancelled is present in the reference signal before it is in the primary signal, the system is causal and successful adaptation will be achieved. This is seen in Eq. (1). As long as the number of filter weights is sufficient to encompass the time delay between the reference and primary signal (in number of samples), the noise present in the primary signal will be related to that seen in an earlier sample still present in $y_{n}$. For proof of the concept see [10].

ANC will be implemented by removing the noise from each microphone array channel in the time domain. These "cleaned" signals will then be used in frequency domain processing to assess the effectiveness of the noise removal.

Beamforming. Beamforming in the frequency domain utilizes phase shifts between the microphone array signals to "steer" the focus of the array and thus obtain more detailed sound pressure magnitude information at defined points in space.

The first step in beamforming is to compute the Cross Spectral Matrix (CSM) for the data set to be analyzed [22]. The CSM is formed from the Fast Fourier Transforms (FFTs) of the data set. The FFTs of two microphones, $m$ and $m^{\prime}$, are denoted $P_{m}(f, \mathrm{~T})$ and $P_{m}(f, \mathrm{~T})$ and are formed from the microphones' time records $p_{m}(\mathrm{t})$ and $\left.p_{m}{ }^{\prime} \mathrm{t}\right)$. The frequencies, $f$, at which the transforms are defined are determined by the bandwidth, $\Delta f=1 / \mathrm{T}(\mathrm{Hz})$, where $\mathrm{T}$ is the data block length. A CSM element, as a function of frequency, is defined

$$
G_{m m^{\prime}}(f)=\frac{2}{K w_{s} \mathrm{~T}} \sum_{k=1}^{K}\left[P_{m k}^{*}(f, \mathrm{~T}) P_{m^{\prime} k}(f, \mathrm{~T})\right]
$$

The total record length is $T_{\text {tot }}=T K$. The summation is multiplied by two because it is a one-sided cross-spectrum. It is then divided by the data block length $T$ to normalize the FFT output and the number of block averages $K$ to get a mean value across the data blocks. The term $w_{s}$ is a weighting constant used when a weighted window is implemented. The star on the first transform denotes the complex conjugate. The CSM element is a complex spectrum. The full CSM for $M$ array microphones is a Hermitian matrix and is given as

$$
\widehat{G}=\left[\begin{array}{cccc}
G_{11} & G_{12} & \cdots & G_{1 M} \\
G_{12}^{*} & G_{22} & & \\
\vdots & & \ddots & \\
G_{1 M}^{*} & & & G_{M M}
\end{array}\right]
$$

The beamforming uses the CSM to electronically "steer" to positions in space defined by the user. For the work done here, the array was one dimensional and the grid points were located along a line that bisected the sound source. In order to steer to grid points on the line, vectors must be calculated between each array microphone and the grid point being steered to. The steering vector for microphone $m$ is

$$
e_{m}=\left(\frac{r_{m}}{r_{c}}\right) e^{j 2 \pi f \tau_{m}}
$$

where $r_{m}$ is the straight line distance from microphone $m$ to the grid point, $r_{c}$ denotes the straight line distance from the center microphone to the grid point, and $\tau_{m}$ is the propagation time for sound to travel between microphone $m$ and the grid point. The term $\left(\frac{r_{m}}{r_{c}}\right)$ is included to normalize the distance dependent amplitude of the steering vector to that of the center array microphone. The steering vector matrix, size $M x 1$, is

$$
\hat{e}=\operatorname{col}\left[\begin{array}{llll}
e_{1} & e_{2} & \cdots & e_{M}
\end{array}\right]
$$


The steering vectors, being complex, will phase shift the contributions from each microphone in the array to allow for constructive summing at the chosen grid point.

Finally, the array's response is given as an output power spectrum

$$
S(\hat{e})=\frac{\hat{e}^{T} \hat{G} \hat{e}}{M^{2}}
$$

The response has units of mean-pressure-squared vs. frequency bandwidth. Dividing by the total number of microphones squared normalizes the response to that of a single microphone level. The superscript $T$ is taking the complex transpose of the steering vector matrix.

DAMAS. The Deconvolution for the Mapping of Acoustic Sources [22] will be used to provide further detail of the sound source under study in the presence of noise. This technique uses the beamform response as a precursor and removes microphone array characteristics (point spread function) to provide an estimation of only the sound source strength that exists in the scanning region. The technique uses an iterative relaxation-type solver to provide non-ambiguous, enhanced resolution presentations of sound sources and is discussed in more detail in [22].

\section{Experimental Setup}

A small anechoic chamber housed at NASA Langley Research Center (LaRC) was used for the test area. Approximate dimensions of the room are $97 \times 77 \times 81$ inches (length/width/height), wedge tip-to-tip.
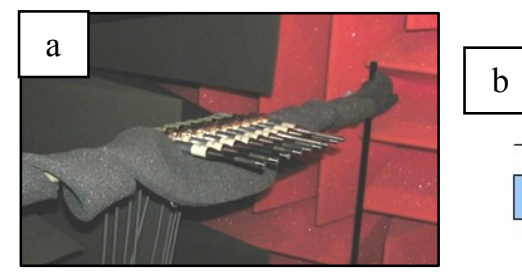

Figure 3. (a) Linear microphone array, and (b) Microphone spacing.

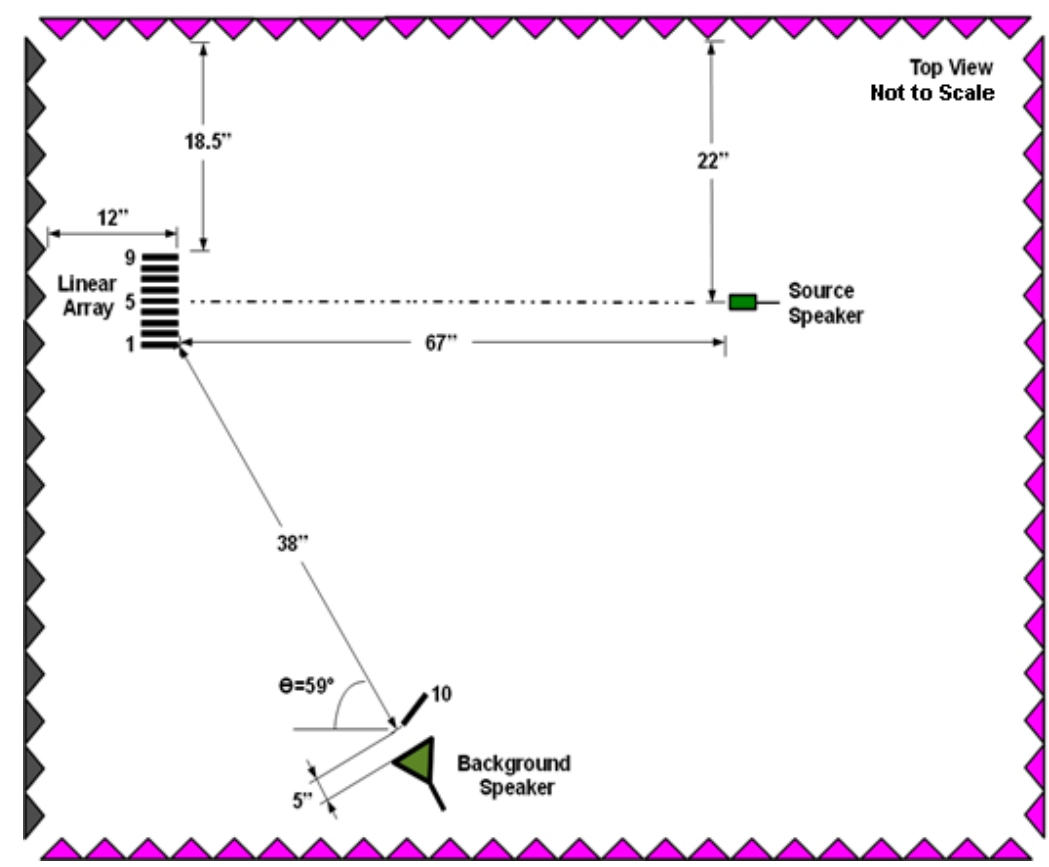

Figure 4. ANC test setup.

Nine B\&K quarter-inch free-field microphones were used to construct a linear array using metal support rods with base stands, as shown in Fig. 3a. The desired microphone spacing was 1" between diaphragm centers; actual spacing differed slightly due to equipment limitations (see Fig. 3b for exact dimensions).

Since the microphone array is linear and configured broadside towards the primary source, its sensing ability lies only along a line parallel with the array at the same vertical height. Thus, the array, source speaker, and background speaker were all placed in the same plane parallel to the floor, at a distance of 46.5" above the floor to ensure that the speaker was high enough above the wedges to avoid low frequency near-field reflections. Refer to Fig. 4 for a diagram of the setup. The source speaker was perpendicular to the array at a distance of 67". The background speaker was at an angle of $59^{\circ}$ (top view) to the array and a distance of 43 " from microphone 1. A reference microphone, labeled 10 , was situated a distance of 5" away from the diaphragm center of the background speaker with its rear facing the source speaker to limit the source speaker's influence on the microphone diaphragm. 

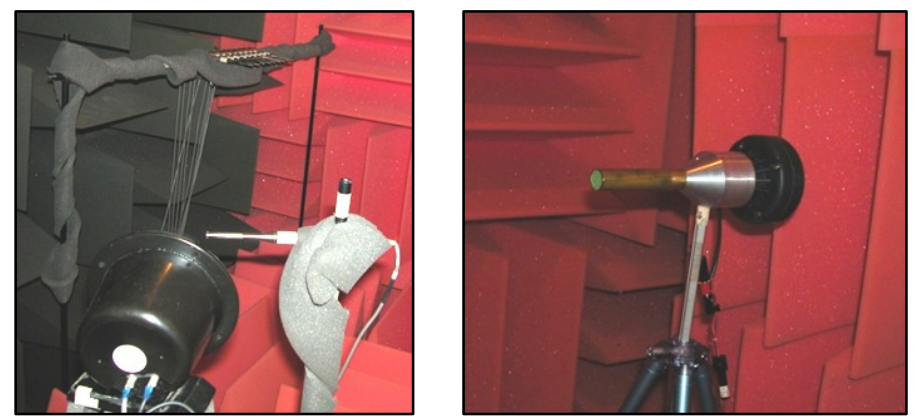

Figure 5. a) ANC setup picture with background speaker and reference microphone in the near-field and array in the far-field, and b) Point source speaker with copper extension tube installed.

A Pioneer 5.25" Cup Midrange background speaker is pictured in the foreground of Fig. 5a. The reference microphone (10) is situated to its right and the microphone array is in the background. A custom source speaker with a Selenium Compression Driver was used as the point source (desired) and is pictured in Fig. 5b. The point source speaker was fitted with a copper extension tube to further simulate an ideal point source and a foam insert was placed in the end of the tube to reduce high frequency reflections. A pink noise generator was used to drive the point source speaker and a white noise generator was used to power the background speaker.

\section{Results}

This section presents example test data that was processed utilizing the signal processing techniques discussed in section II. The frequency resolution for the data presentations is $20 \mathrm{~Hz}$. The microphone signals were high-pass filtered at $400 \mathrm{~Hz}$ to avoid any low-frequency reflections present in the chamber.

Table 1 gives parameters for the cases analyzed in this study in order to investigate the potential and limitations associated with ANC in microphone array applications. The first two use microphone 10 (see Fig. 4) as the reference channel to cancel noise at the array microphones. Microphone 10 gives the best estimate of the noise because of its proximity to the background speaker. The first case examines results at a low SNR. This results in high coherence between microphone 10 and the array microphones. The second case is for a higher SNR acquisition. The coherence between microphone 10 and the array microphones drops as a result of the increased SNR at the array. The third and fourth cases follow the same pattern. However, microphone 5 is used as the reference channel to cancel the noise on the array microphones. Microphone 5, being the center array microphone, gives an average of the noise sensed over the array.

Table 1. Test Cases.

\begin{tabular}{|c|c|c|c|c|c|c|}
\hline & & & & & \multicolumn{2}{|c|}{ Narrowband Coherence } \\
\hline Case & Acquisition & $\begin{array}{c}\text { Average SNR, } \\
\mathbf{0 . 5 - 9 . 5} \mathbf{~ k H z}(\mathbf{d B})\end{array}$ & $\begin{array}{c}\text { Narrowband } \\
\text { Frequency (kHz) }\end{array}$ & $\begin{array}{c}\text { Narrowband } \\
\text { SNR (dB) }\end{array}$ & $\begin{array}{c}\text { Mic 10 - } \\
\text { Array }\end{array}$ & $\begin{array}{c}\text { Mic 5 - } \\
\text { Array }\end{array}$ \\
\hline 1 & a & -19.9 & 6.3 & -29.2 & 0.95 & -- \\
\hline 2 & b & -6.5 & 8.4 & -3.6 & 0.43 & -- \\
\hline 3 & a & -19.9 & 6.3 & -29.2 & -- & 0.99 \\
\hline 4 & b & -6.5 & 8.4 & -3.6 & -- & 0.43 \\
\hline
\end{tabular}

Note that while the low SNR cases pose the largest signal recovery in decibels (dB), the high coherence between the reference and array microphones will be beneficial to ANC recovery. This is because attenuation as a function of coherence $\left(\gamma^{2}\right)[23,24]$ is calculated as

$$
\text { attenuation }(d B)=10 \times \log _{10}\left(1-\gamma^{2}\right)
$$

For each case, the noise attenuation results using ANC will be compared to spectral subtraction using autospectra. Then, array processing techniques (beamforming and DAMAS, see section II) will be used to compare the noise attenuation results from ANC with CSM subtraction. 
Case 1. Figure 6 displays autospectra from the center array microphone. For all cases, the sampling frequency used was $20 \mathrm{kHz}$. The digital antialiasing filter on the data acquisition system was set to $9.7 \mathrm{kHz}$; the low-pass filter rolls off sharply: by the Nyquist frequency $(10 \mathrm{kHz})$ the reduction is $-10 \mathrm{~dB}$, and by $10.5 \mathrm{kHz}$ the reduction falls to $50 \mathrm{~dB}$ with zero phase error. The drop-off due to the low-pass filter will be seen in the subsequent autospectra. Note that the data are plotted as power spectra and thus specific to the acquisition parameters. The blue line corresponds to the acquisition when both the point source and background speakers were operated simultaneously. The average SNR between the two over the frequency range $0.5-9.5 \mathrm{kHz}$ is $-19.9 \mathrm{~dB}$. The noise floor (all equipment off) of the room is plotted in light blue for reference. The desired outcome of the noise attenuation processing is to recover the point source spectrum (red) from the combined spectrum (source plus noise, blue).

The noise attenuation results from spectral subtraction (see section I) are shown in black. The choppiness of the signal is due to the $20 \mathrm{~Hz}$ bandwidth. Large dips in the signal correspond to frequencies at which the value of the acquisition of just the background noise speaker operating (not shown) approaches that of the combined acquisition (blue). It is seen that that an average attenuation from the combined spectrum of $11 \mathrm{~dB}$ is achieved $(0.5-9.5 \mathrm{kHz})$. Again, the point source spectrum (red) is the signal to recover. Even with the achieved attenuation, the spectral subtraction data are still $6 \mathrm{~dB}$ or more above the point source spectrum.

The noise attenuation data using ANC with microphone 10 as the background noise reference are plotted in bright green. The results give almost perfect attenuation across the $0.5-9.5 \mathrm{kHz}$ frequency band, providing an excellent recovery of the point source signal. This is due to two factors: i) high coherence present between the reference and array microphones, and ii) the reference microphone being dominated by the background noise due to its proximity to the background speaker. The second factor allows the reference microphone to obtain a very good estimate of the background noise signal free of contamination from the point source signal. This is important because microphone 10 is not shielded from point source speaker radiation. It experiences similar levels to the array microphones when just the point source is operated. When both the point source and background speakers are operated, microphone 10's level is an average of $20 \mathrm{~dB}$ greater than the array microphones, which yields almost 40 $\mathrm{dB}$ greater $(0.5-9.5 \mathrm{kHz})$ than the point source alone for this case. This difference in level effectively "shields" microphone 10 from point source influence.

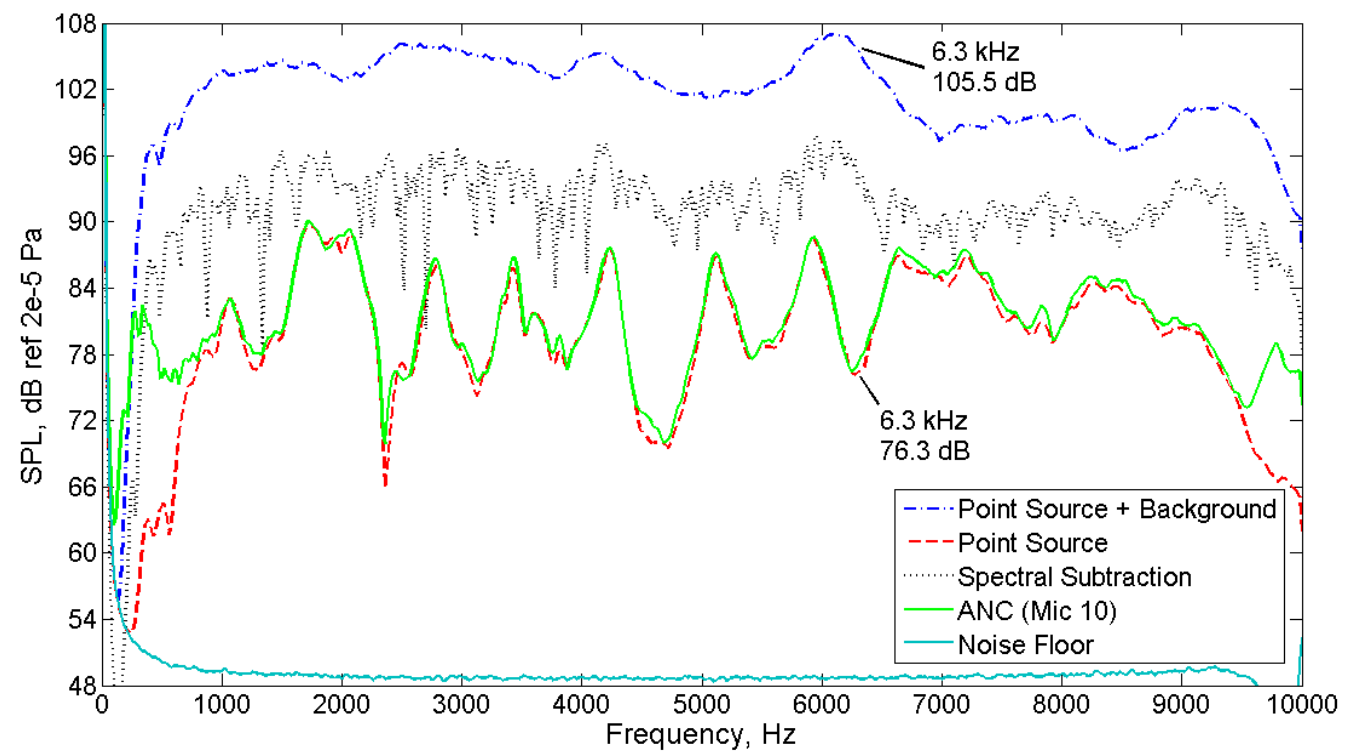

Figure 6. Center array microphone autospectra for Case 1.

Referencing section II, beamforming is an array technique which calculates sound pressure magnitude at distinct points in space. The points in space are user defined with a grid. The grid location is chosen to provide details of the sound source under study, in this case the point source speaker. Thus, a $4 \mathrm{ft}$ horizontal line at the height of the point source speaker with its center at the speaker location was chosen as the one dimensional scanning grid over which to perform the beamforming. Note that beamforming as a technique already attenuates noise as uncorrelated terms in the CSM tend to zero in the statistical limit [21]. Therefore, beamforming offers SNR improvements over single microphone spectra, and thus CSM subtraction an improvement over spectral subtraction. 
Figure 7 gives the beamform responses at $6.3 \mathrm{kHz}$. Criteria from [22] for a reasonable resolution range were used. The color conventions were kept the same as Fig. 6 for continuity and are used throughout. The desired outcome of the noise attenuation processing is to recover the point source beamform (red) from the point source plus background noise beamform (blue). The combined point source and background speaker acquisition beamform is clearly distorted. At almost $30 \mathrm{~dB}$ down (Fig. 6), the small (9 microphone) array has difficulty recovering the point source strength $(\mathrm{x}=0 \mathrm{ft})$ or lobe structure from the combined acquisition. Note that a larger array would have more accurate desired signal recovery under the same conditions due to the array SNR gain [21]

$$
\operatorname{array} \operatorname{gain}(d B)=10 \times \log _{10}(\# \text { of array microphones })
$$

The black line corresponds to the noise attenuated beamform obtained through CSM subtraction (see sections I and II). While it matches the point source level (slightly to the right of $\mathrm{x}=0 \mathrm{ft}$ ), its distorted sidelobe structure is similar to that of the combined acquisition. The green line gives the noise attenuated beamform using ANC with microphone 10 as the reference signal. It matches the point source beamform well at the source location $(\mathrm{x}=0 \mathrm{ft})$ and has similar sidelobe structure, indicating that the point source magnitude is recovered and phase preserved correctly during the ANC process.

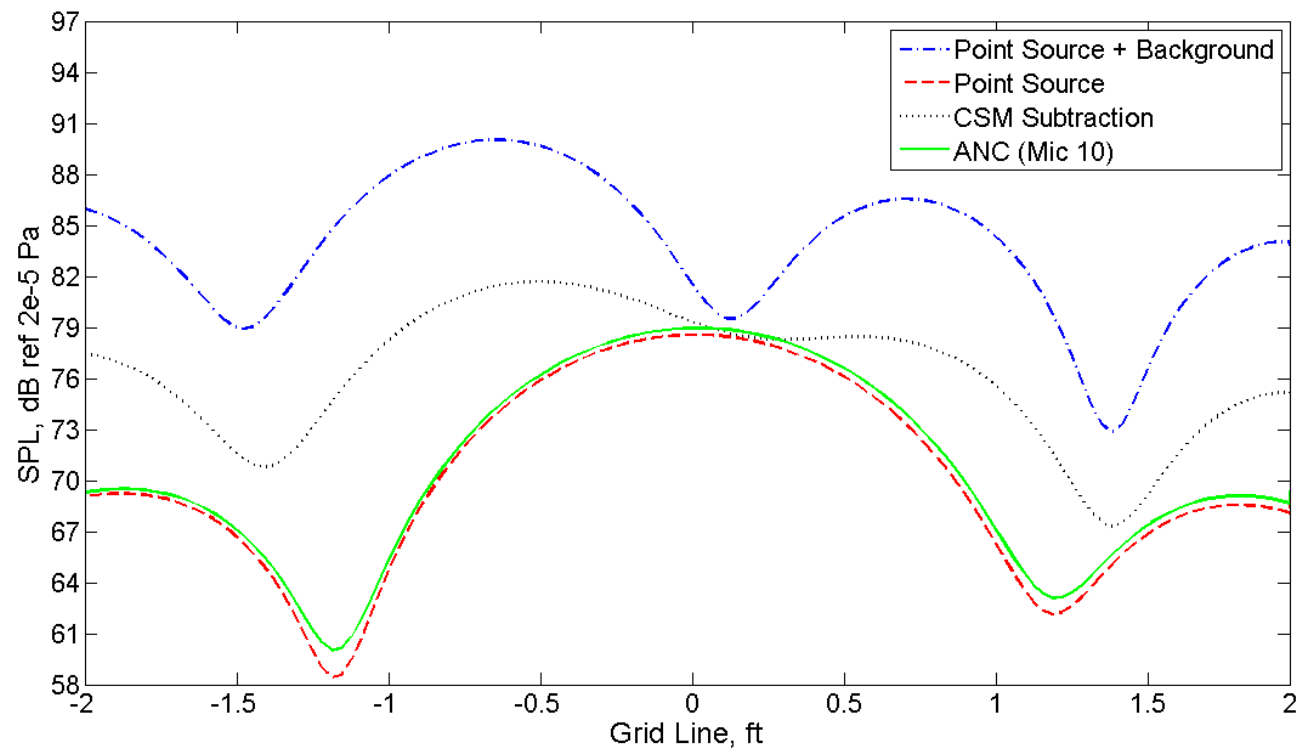

Figure 7. Beamform results for Case $1(6.3 \mathrm{kHz}, \mathrm{SNR}=-29.2 \mathrm{~dB})$.

Deconvolution using DAMAS [22] removes microphone array characteristics from the beamform response to provide an estimation of only the sound source strength that exists in the scanning grid. For instance, if the point source speaker were a dot in space at $\mathrm{x}=0 \mathrm{ft}$ operating at $79 \mathrm{~dB}$, an ideal DAMAS deconvolution would converge through an iterative process on one point at $\mathrm{x}=0 \mathrm{ft}$ with a level of $79 \mathrm{~dB}$. All the rest of the grid point levels would tend towards negative infinity, because the point source speaker is the only sound source located in the scanning region. This deconvolved beamform would represent an enhanced resolution sound map indicating the exact location in the scanning grid and sound pressure level (SPL) of the point source speaker.

Referring to the beamform responses given in Fig. 7, a resolution range for the DAMAS results was chosen to be $65-89 \mathrm{~dB}$. Note that experimental results will not fall to $0 \mathrm{~dB}$ where no sound sources exist in the beamform grid. This lower SPL bound will illustrate that the deconvolution eliminates the sidelobe structure present in the beamform responses. This presentation choice will be used throughout.

Figure 8 displays the DAMAS results processed from the beamform responses of Fig. 7. Convergence was reached after 45 iterations. The goal of the noise attenuation processing is to recover the point source deconvolution map from the combined point source and background data. The combined map displays four "source" peaks over the grid. All are erroneous in location and level. This can be stated because the point source map identifies the correct location and level of the point source speaker, the only sound source located in the scanning grid. The deconvolved map from the beamform using CSM subtraction also fails to locate the point source speaker in location or level. The 
DAMAS results from the ANC beamform response converge on a deconvolved map that correctly locates the point source speaker location and estimates it strength to within $0.3 \mathrm{~dB}$.

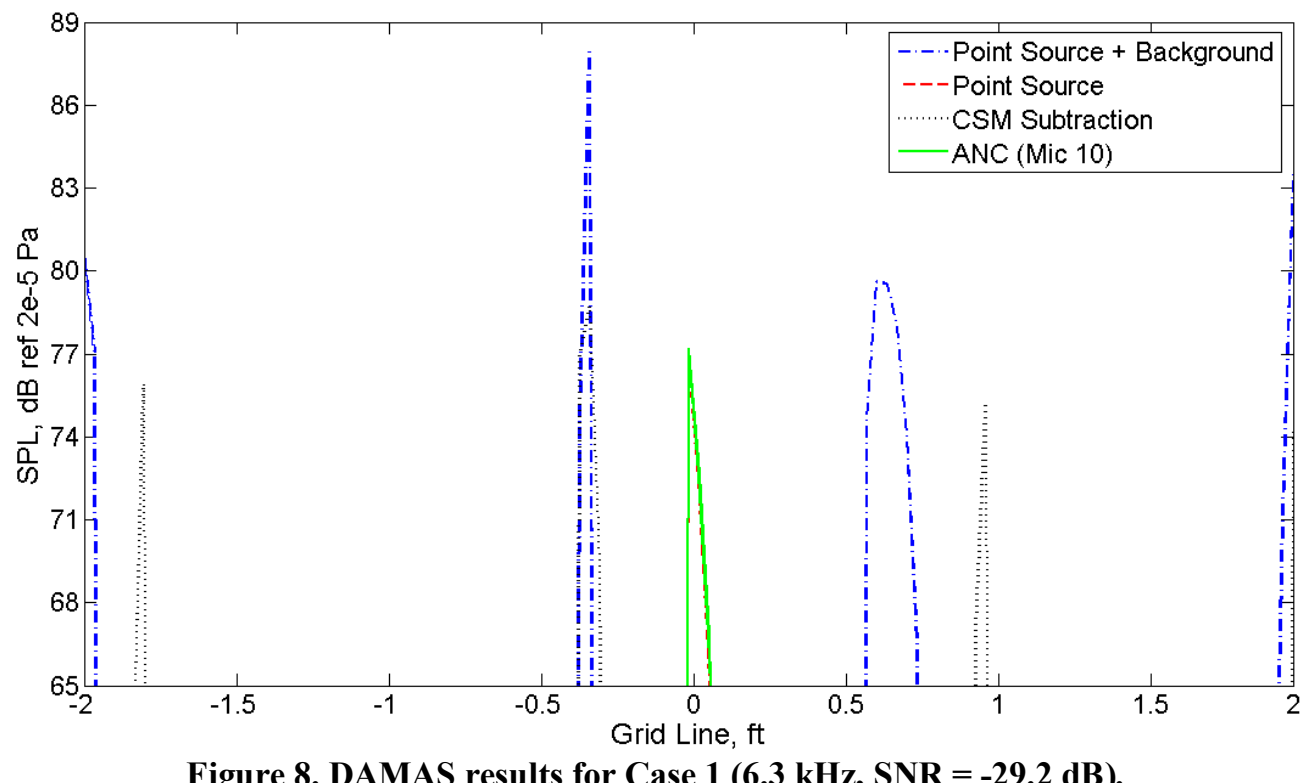

Figure 8. DAMAS results for Case $1(6.3 \mathrm{kHz}, \mathrm{SNR}=-29.2 \mathrm{~dB})$.

Case 2. Figure 9 presents the autospectra from the center array microphone. The average SNR between combined and point source spectrum over the frequency range $0.5-9.5 \mathrm{kHz}$ is $-6.5 \mathrm{~dB}$. The spectral subtraction results are seen to recover the point source spectrum more accurately due to the higher SNR (with respect to Case 1). Loss of recovery accuracy occurs at frequencies where the SNR decreases. The ANC results using microphone 10 again give almost perfect attenuation across the $0.5-9.5 \mathrm{kHz}$ frequency band. Note that at the frequency chosen for narrowband analysis $(8.4 \mathrm{kHz})$, the average coherence between microphone 10 and the array microphones is 0.43 . Even with this low coherence the ANC recovered spectrum is within $0.1 \mathrm{~dB}$ of the point source level. This is attributed to the relatively high SNR and again because microphone 10's signal is dominated by the background noise, due to its proximity to the background speaker, which provides an accurate estimate of the noise to be cancelled from the array signals.

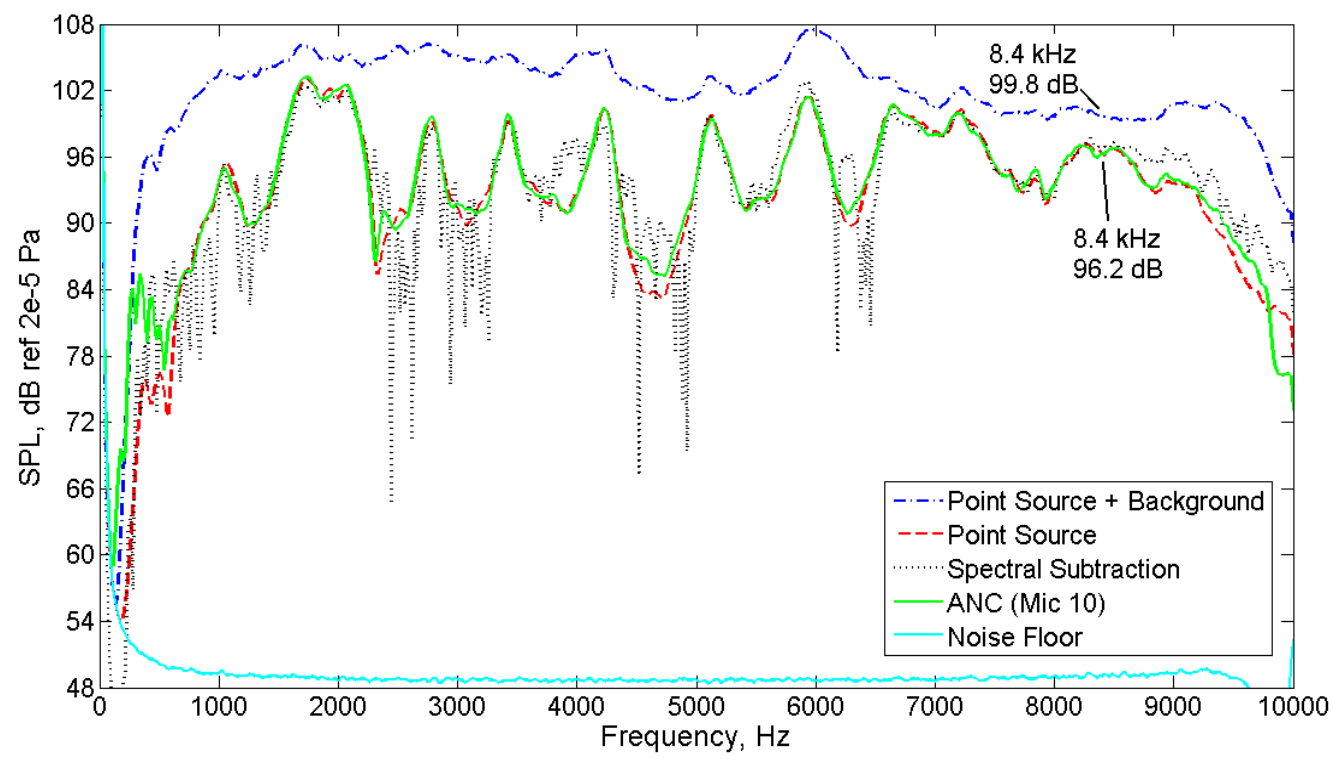

Figure 9. Center array microphone autospectra for Case 2.

American Institute of Aeronautics and Astronautics 
Figure 10 gives the beamforming results at $8.4 \mathrm{kHz}$ for Case 2. Due to the increased SNR (with respect to Case $1)$, the beamform of the combined acquisition matches the point source only results at the source position $(\mathrm{x}=0 \mathrm{ft})$ and has similar sidelobe structure. This is achieved without any noise attenuation processing and provides evidence that beamforming as a technique attenuates noise due to uncorrelated noise being cancelled upon forming the CSM. Both the processed, noise attenuated beamform responses, CSM subtraction and ANC using microphone 10, match the point source level at the speaker location and have very similar sidelobe structures. At this SNR, both methods are effective at recovering the point source beamform response. The low coherence $(0.43)$ present at this frequency did not hinder the ANC recovery.

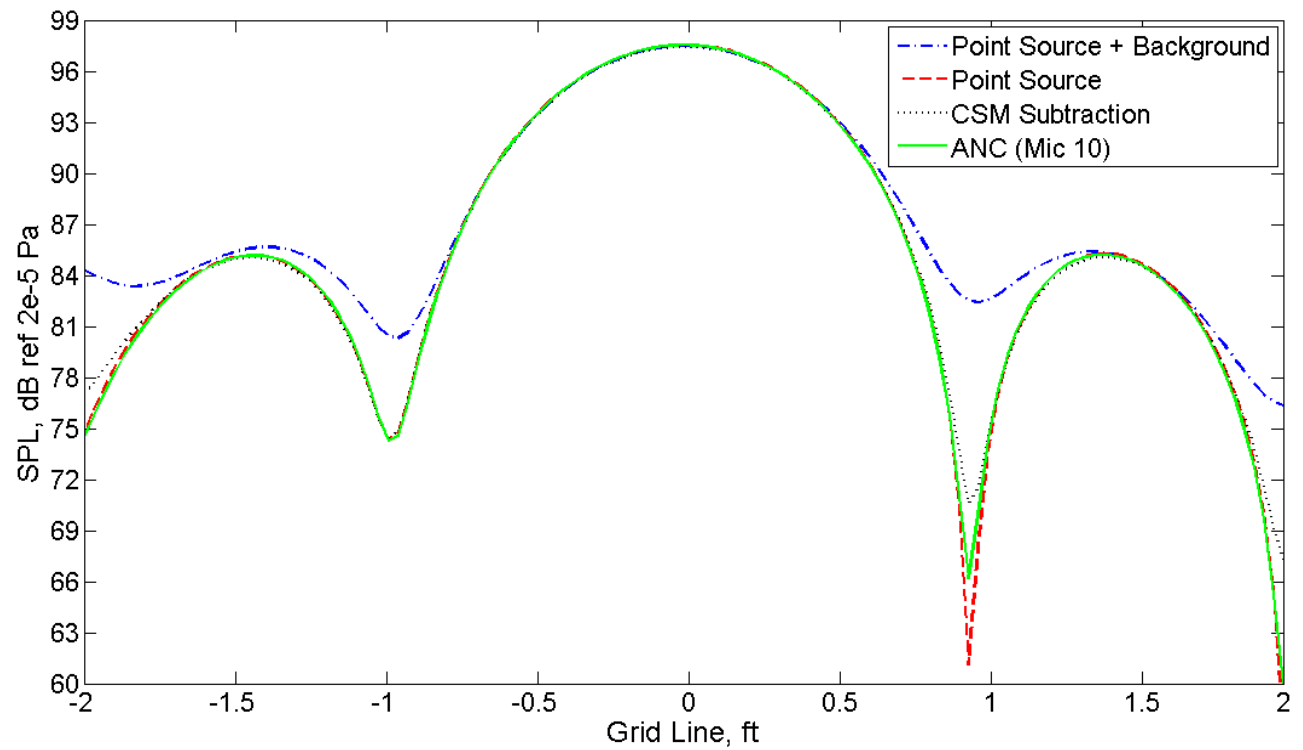

Figure 10. Beamform results for Case $2(8.4 \mathrm{kHz}, \mathrm{SNR}=\mathbf{- 3 . 6} \mathrm{dB})$.

Figure 11 displays the DAMAS results processed from the beamform responses of Fig. 10. Convergence was reached after 58 iterations. Although the combined map displays various low-level "sources" they are $14 \mathrm{~dB}$ or more below the point source level $(\mathrm{x}=0 \mathrm{ft})$. Its largest peak is located accurately at the point source speaker location and it estimates the source strength to within $0.7 \mathrm{~dB}$. The DAMAS results from the CSM subtraction and ANC beamform responses converge at the correct point source speaker location and estimate its level to within $0.1 \mathrm{~dB}$.

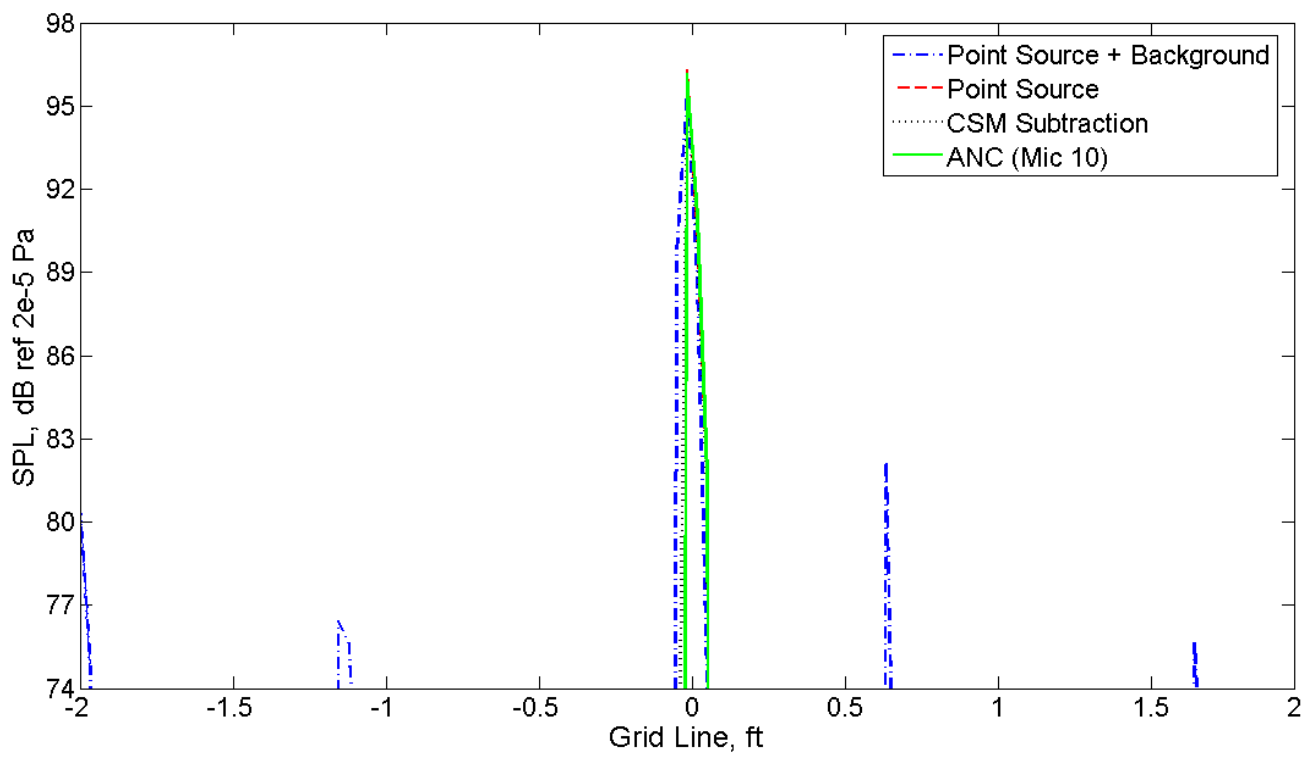

Figure 11. DAMAS results for Case $2(8.4 \mathrm{kHz}, \mathrm{SNR}=-3.6 \mathrm{~dB})$. 
Cases 3 and 4 take a difference approach to the ANC processing due to the fact that array microphone 5 is used as the background noise reference signal. As microphone 5 is directly exposed to the point source speaker radiation and records levels on the order of the other array mics, its signal should not be adapted during the noise removal filtering. This is due to the concern that the desired signal will be cancelled along with the noise from the channels being processed with microphone 5 as the noise reference [12], because they are both exposed to point source radiation at similar magnitudes (contrast this with microphone 10). Thus, the ANC filter weights are converged using the acquisition of only the background noise speaker operating, then saved to process (with zero adaptation, $\mu$ $=0$ ) the signals obtained during the combined (point source plus background noise) acquisition. If the background noise were exactly repeatable, using the saved weights from background noise acquisition processing and zero adaptation would achieve exact cancellation of the noise. This is not the case as random white noise was used as the background noise input. The ANC filter adapts its weights based on time-domain transducer signals. While the background noise from one acquisition to the next is spectrally similar, no two randomly generated time signals are the same. Thus, the weights used from the background only acquisition will be good estimates of the weights required for background noise cancellation in the combined case, but not exact. As adaptation is frozen $(\mu=0$, weights are not updated), error is introduced in recovery. It is noted that time-domain cancellation, in the limit, will always perform better than spectral cancellation because it is exactly cancelling the noise at each time step in the processing. Spectral cancellation relies on statistical approximation and therefore slight variations in the noise are not accounted for and must be averaged to produce an estimate of the noise.

Case 3. Figure 12 presents the array averaged autospectra for this case. The data are from the same acquisition as Case 1. For Cases 3 and 4 the autospectra are array averaged. The same spectral subtraction performance from Case 1 is seen here: an average attenuation of $11 \mathrm{~dB}$ is achieved but the data are still $6 \mathrm{~dB}$ or above the point source spectrum $(0.5-9.5 \mathrm{kHz})$.

The noise attenuation data using ANC with microphone 5 as the background noise reference are plotted in bright green. The results give significant attenuation across the $0.5-9.5 \mathrm{kHz}$ frequency band, but are an average $3 \mathrm{~dB}$ above the point source spectrum (the desired signal to recover). Although the point source autospectrum recovery is not as accurate using microphone 5 as the noise reference (with respect to microphone 10), its performance is significantly better than spectral subtraction for this low SNR acquisition. Again, note that this recovery was obtained without adaptation. Only the saved filter weights from adapting the signals with the background only acquisition data were used in the processing. This procedure is similar to establishing a transfer function of the background noise between the reference and array microphones, then using this "transfer function" to remove noise from combined acquisition data. And in this case, as one of the array microphones was used as a reference, no other "reference" signals are needed (contrast with methodology from Cases 1 and 2 where a separately located reference microphone is used).

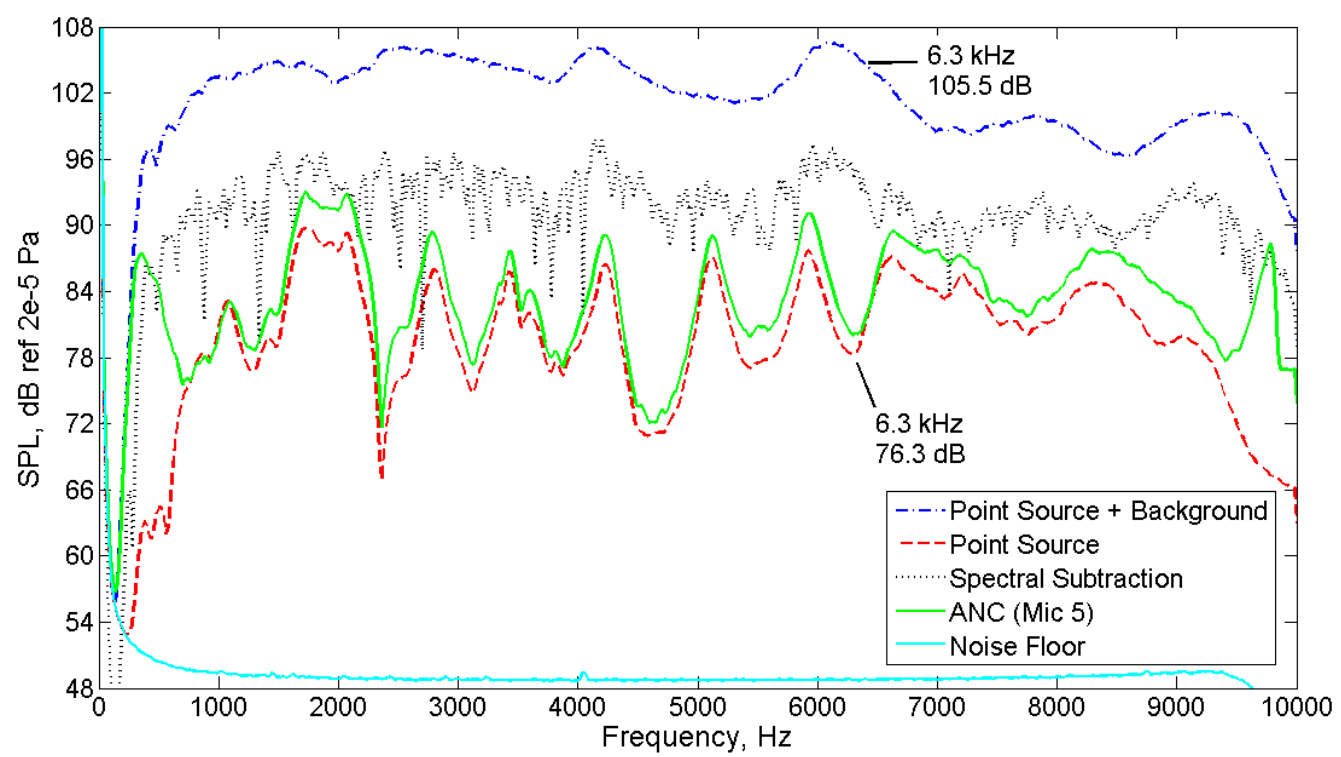

Figure 12. Array averaged autospectra for Case 3. 
Figure 13 gives the beamform responses for this case at $6.3 \mathrm{kHz}$. Note because microphone 5 is used as reference it cannot be used as an array microphone for beamforming. This is because another array microphone would have to be used as a reference to attenuate the noise on microphone 5. This would lead to slightly different results not consistent with the other array microphones, because slightly different noise would be attenuated. Therefore, only array microphones 1-4 and 6-9 were used for beamforming and subsequent DAMAS processing. This applies to both Case 3 and 4. The level and shape discrepancies between the beamform responses from Cases 1 $\& 2$ vs. $3 \& 4$ are due to this. The combined point source and background speaker acquisition beamform, using now only 8 microphones, is even more distorted than that of Fig. 7. This is noted here because these are the same acquisitions. The spectral subtraction beamforming achieves a $7 \mathrm{~dB}$ reduction at the point source location $(\mathrm{x}=0 \mathrm{ft})$ but clearly fails at recovering the point source response. The ANC beamform response using microphone 5 recovers the point source level at the speaker location to within $1 \mathrm{~dB}$ and has a similar sidelobe structure. The conclusion drawn from Fig. 12 holds here: while ANC recovery using microphone 5 as reference is not as accurate as using microphone 10, at this SNR it outperforms CSM subtraction and recovers the point source strength to within $1 \mathrm{~dB}$.

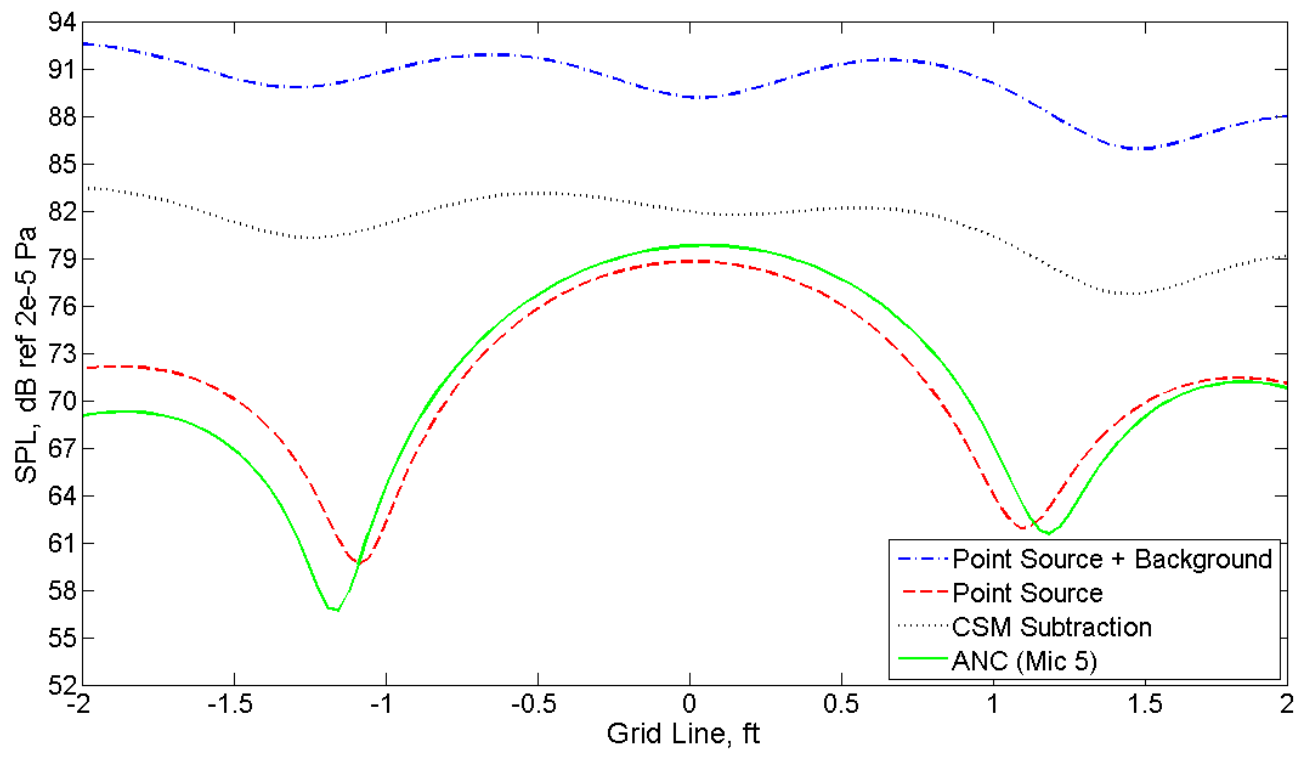

Figure 13. Beamform results for Case $3(6.3 \mathrm{kHz}, \mathrm{SNR}=\mathbf{- 2 9 . 2} \mathrm{dB})$.

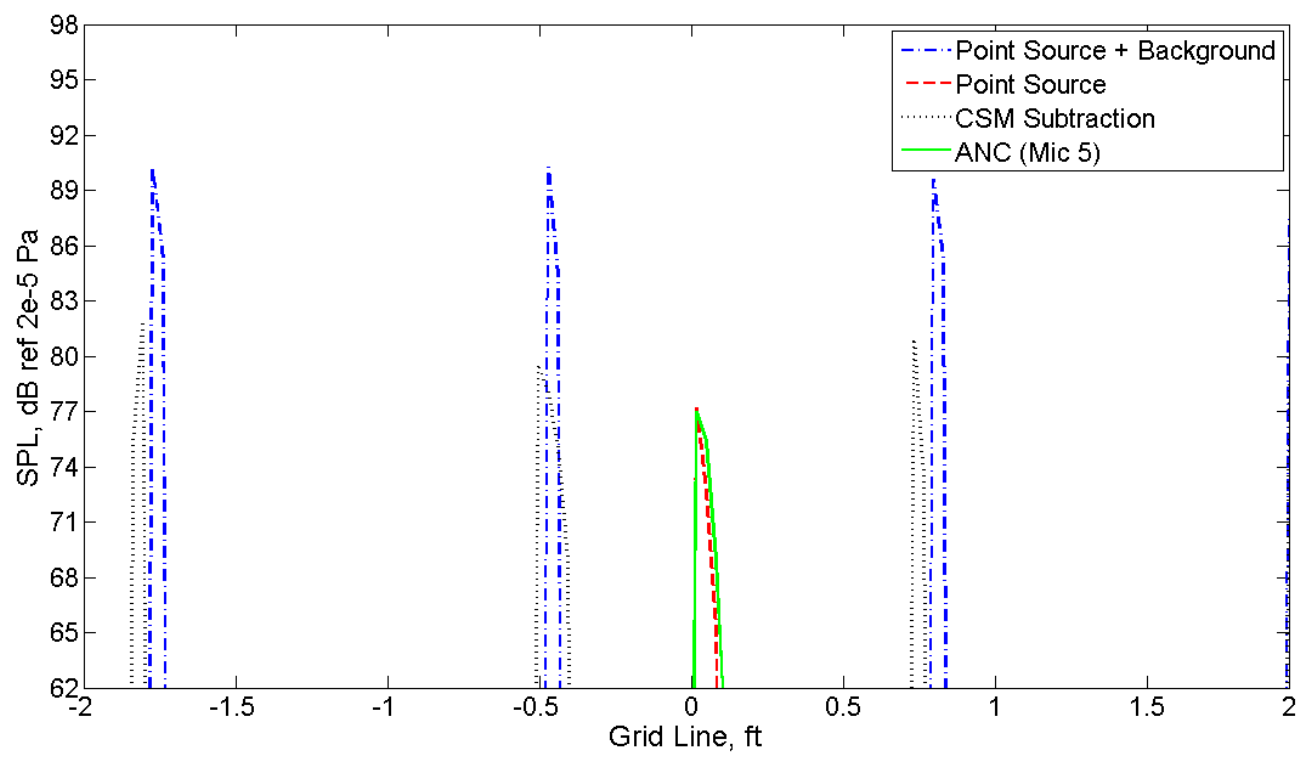

Figure 14. DAMAS results for Case $3(6.3 \mathrm{kHz}, \mathrm{SNR}=-29.2 \mathrm{~dB})$. 
Figure 14 gives the corresponding deconvolved sound map for Fig. 13. Convergence was reached after 45 iterations. The combined map displays four erroneous "source" peaks over the grid. The CSM subtraction results display three. All erroneous peaks are greater than the point source level. The deconvolved map using ANC with microphone 5 as reference correctly identifies the point source speaker location and estimates its strength to within $0.1 \mathrm{~dB}$. Note that compared to Case 1, Case 3 ANC processing yielded less accurate autospectra and beamforming results. However, the ANC beamforming results for Case 3 were accurate enough to yield the same accuracy in deconvolution as Case 1 ANC results (compare Figs. 8 and 14).

Case 4. This final case employs the same processing technique as Case 3 on the data from the higher SNR and lower coherence acquisition.

Figure 15 presents the array averaged autospectra for this case. The data are from the same acquisition as Case 2. The same spectral subtraction performance from Case 2 is repeated: the point source spectrum is recovered accurately where SNRs are higher, and loses accuracy with decreasing SNR. The ANC processing recovers the point source data to within $2 \mathrm{~dB}$ across most of the $0.5-9.5 \mathrm{kHz}$ frequency range. The ANC processing outperforms spectral subtraction accuracy wise. As with Case 3 compared to 1, the Case 4 ANC recovery is not as accurate as the ANC recovery of Case 2 .

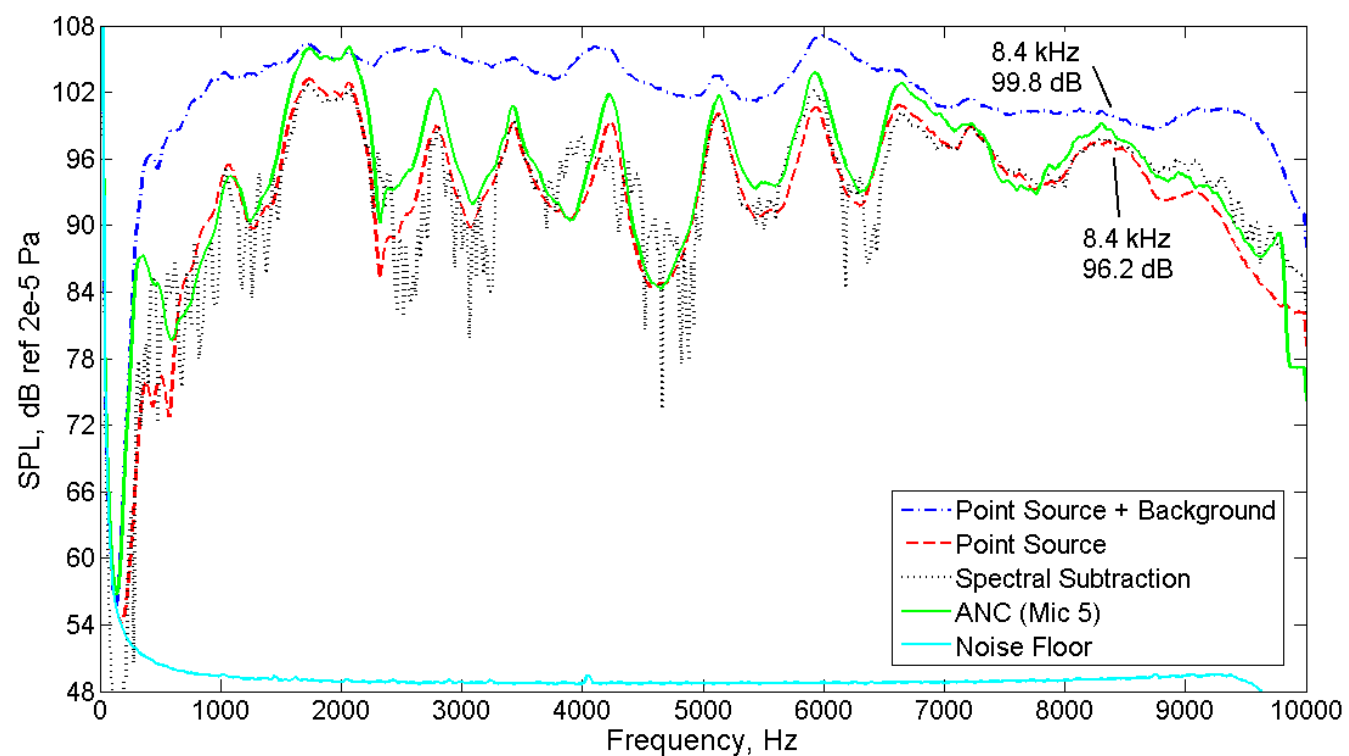

Figure 15. Array averaged autospectra for Case 4.

Figure 16 presents the Case 4 beamform responses. Due to the high SNR, the beamforming is able to accurately recover the point source spectrum from the combined acquisition without any noise attenuation processing. CSM subtraction further improves the recovery accuracy. ANC processing is even less accurate than the combined acquisition beamform response. This is due to the low coherence (0.43) present between microphone 5 and the rest of the array channels at this frequency and because filter weight adaptation was frozen.

The deconvolved beamform maps are given in Fig. 17. Although the combined acquisition DAMAS result contains two erroneous peaks $(\mathrm{x}=-1.9,0.7)$, they are more than $14 \mathrm{~dB}$ from the correct point source level at $\mathrm{x}=0 \mathrm{ft}$. It estimates the point source strength to within $1.6 \mathrm{~dB}$ with no noise attenuation processing. The beamform (Fig. 16) and its deconvolution display the noise attenuation capabilities of these array processing techniques at this SNR. The deconvolved map using CSM subtraction accurately predicts the point source location and level to within $0.02 \mathrm{~dB}$. This is excellent recovery at this SNR. As with the beamform response (Fig. 16), the ANC results are the least accurate. The predicted point source speaker location is 0.4 " to the right of the true location. Its level was predicted $2.8 \mathrm{~dB}$ higher than the actual. 


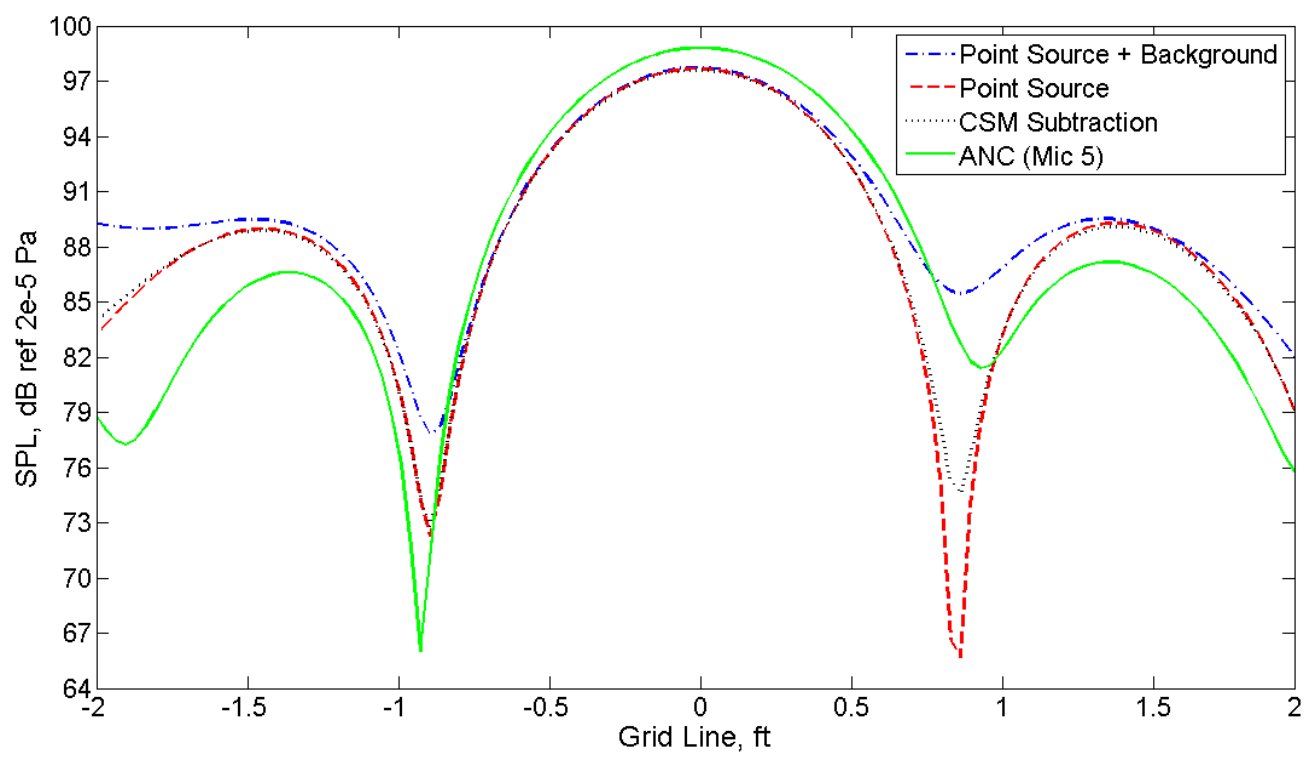

Figure 16. Beamform results for Case $4(8.4 \mathrm{kHz}, \mathrm{SNR}=-\mathbf{- 3 . 6} \mathrm{dB})$.

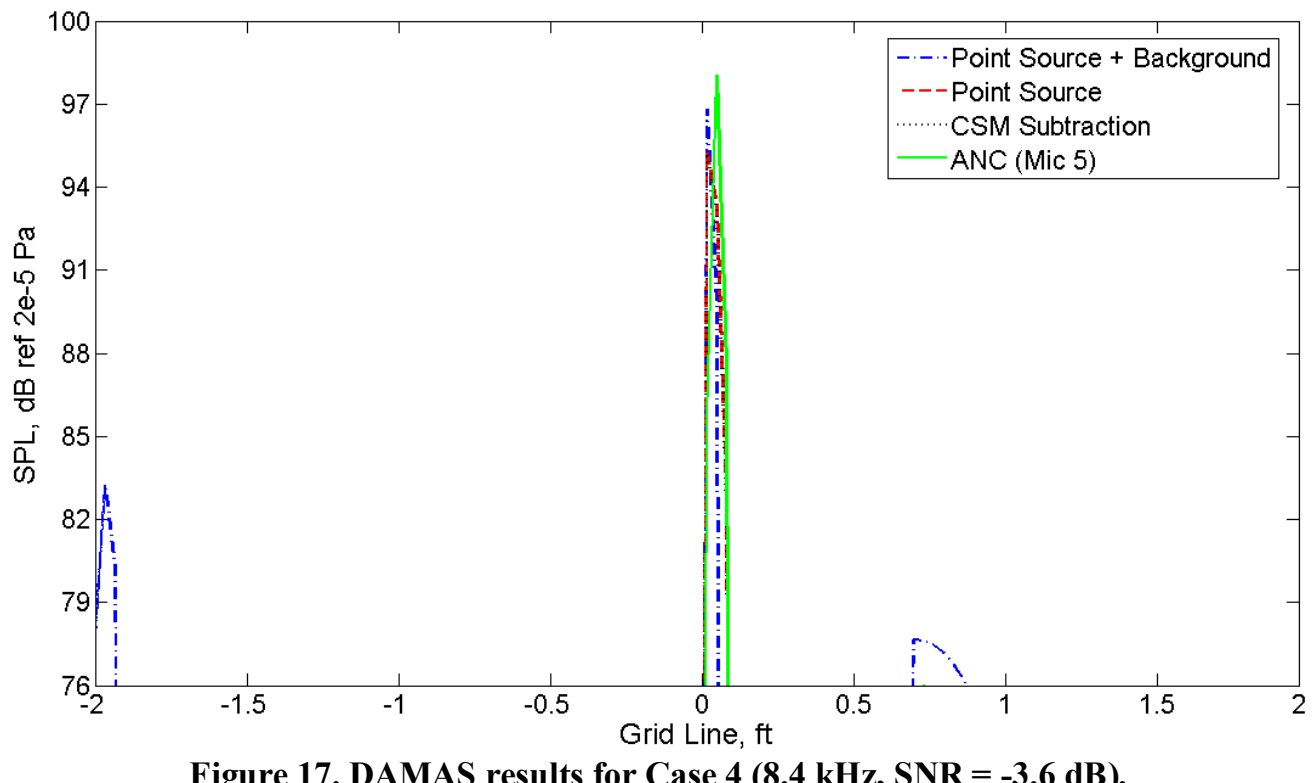

The Case 4 results demonstrate that at frequencies with relatively high SNRs the spectral and CSM subtraction methods provide accurate recovery of the point source level. The array technique of beamforming (without noise attenuation processing) is able to recover the point source magnitude and lobe structure. Subsequent deconvolution using DAMAS correctly estimated the point source speaker location with little error (Fig. 17). CSM subtraction only improved the accuracy of the beamform response and corresponding deconvolution map. Due to low coherence at the narrowband frequency chosen, the ANC results without adaptation using the center array microphone were less accurate than those with higher coherence (Case 3), and using microphone 10 as reference with adaptation (Case 2). 


\section{Conclusions}

The application of Adaptive Noise Cancellation with microphone array signals has been investigated in a simplified setup using a background noise and a point source speaker. The background speaker was intended to produce average Signal-to-Noise Ratios across the frequency band $0.5-9.5 \mathrm{kHz}$ as low as $-20 \mathrm{~dB}$ at the array. A reference microphone was located close to the background noise speaker in order to obtain a nearly ideal acquisition of the background noise.

Two cases investigated the noise attenuation processing at a low SNR and high coherence between the reference and array microphones. Spectral subtraction was insufficient in recovering the point source magnitude over the frequency band analyzed. CSM subtraction also failed at recovering a beamform response accurate enough to yield convergence on the correct point source location and magnitude in subsequent deconvolution using DAMAS. ANC processing using a reference microphone close to the background noise source proved highly accurate in recovering the point source magnitude over the frequency band, beamform response, and corresponding deconvolution map. The ANC processing using the center array microphone as a noise reference and no filter adaptation was also successful in the recovery of the point source magnitude and location, but yielded less accurate results than those processed using the reference microphone close to the background noise source.

Two additional cases analyzed the noise attenuation processing performance under relatively higher SNR and lower coherence conditions. Spectral subtraction yielded accurate recovery of the point source magnitude at frequencies where high SNR was present. Beamforming of the signal plus noise acquisition was sufficient to recover the point source magnitude and location in the deconvolved beamform maps for these cases due to the higher SNR. CSM subtraction only improved this performance. The ANC results using the microphone placed near the background noise source yielded the best results of any of the processing methods. The low coherence did not reduce its recovery accuracy because of its ideal background noise estimate. ANC using the center array microphone yielded the least accurate results due to the low coherence present between the center and array microphones and because the filter adaptation was frozen. However, its recovery was sufficient to locate and predict the point source magnitude in its deconvolved beamform map.

The ANC technique using a reference microphone placed near the background noise source would be effective for localizable noise sources which retain coherence between the reference and array microphones. Using the center array microphone as a reference to attenuate the noise on the other array channels with ANC has more general applications and the possibility of improving upon the current noise attenuation methods. Analysis of the techniques with a more populated microphone array under more realistic test conditions remains to be performed.

\section{Acknowledgments}

The author would like to thank Dr. Noah Schiller and Mr. Jacob Klos for aid with the experimental setup. Taylor Spalt and Chris Fuller are grateful for the funding of their work by NASA under contract NNL08AA00B.

\section{References}

${ }^{1}$ Wambsganss, M.W. and Zaleski, P.L. "Measurements, Interpretation and Characterization of Near Field Flow Noise". ANL-7685, 1970, pp. 112-140.

${ }^{2}$ Wilson, R.J., Jones, B.G., and Roy, R.P. "Measurement Techniques of Stochastic Pressure Fluctuations in Annular Flow". Proc 6th Biennial Symp on Turbulence, Univ. Missouri-Rolla, 1979.

${ }^{3}$ Home, M.P. and Hansen, R.I. "Minimization of Far Field Acoustic Effects in Turbulent Boundary Layer Wall Pressure Fluctuation Experiments”. Proc 7th Symp on Turbulence, University of Missouri-Rolla, 1981, pp. 139-148.

${ }^{4}$ Lauchle, G.C. and Daniels, M.A. "Wall-pressure Fluctuations in Turbulent Pipe Flow”. Phys. Fluids (30), 1987, pp. 30193024.

${ }^{5}$ Simpson, R.L., Ghodbane, M., and McGrath, B.E. "Surface Pressure Fluctuations in a Separating Turbulent Boundary Layer”. Journal of Fluid Mechanics (177), 1987, pp. 167-186.

${ }^{6}$ Agarwal, N.K. and Simpson, R.L. "A New Technique for Obtaining the Turbulent Pressure Spectrum from the Surface Pressure Spectrum". Journal of Sound and Vibration (135), 1989, pp. 346-350.

${ }^{7}$ Naguib, A.M., Gravante, S.P., and Wark, C.E. "Extraction of Turbulent Wall-Pressure Time-Series using an Optimal Filtering Scheme". Experiments in Fluids (22), 1996, pp. 14-22.

${ }^{8}$ Widrow, B., and Hoff Jr, M.E. “Adaptive Switching Circuits”. IRE WESCON Convention Record, Vol. 4, 1960 , pp. 96-104.

${ }^{9}$ Koford, J.S., and Groner, G.F. "The Use of an Adaptive Threshold Element to Design a Linear Optimal Pattern Classifier". IEEE Transactions on Information Theory, Vol. 12, No. 1, 1966, pp. $42-50$.

${ }^{10}$ Widrow, B., Glover Jr., J.R., McCool J.M., Kaunitz, J., Williams, C.S., Hearn, R.H., Zeidler, J.R., Dong Jr., E., and Goodlin, R.C. "Adaptive Noise Cancelling: Principles and Applications". Proceedings of the IEEE, Vol. 63, No. 12, 1975, pp. 1692-1716. 
${ }^{11}$ Boll, S.F. and Pulsipher, D.C. "Suppression of Acoustic Noise in Speech Using Two Microphone Adaptive Noise Cancellation”. IEEE Transactions on Acoustics, Speech, and Signal Processing, Vol. ASSP-28 (6), 1980, pp. $752-753$.

${ }^{12}$ Harrison, W.A., Lim, J.S., and Singer, E. “A New Application of Adaptive Noise Cancellation”. IEEE Transactions on Acoustics, Speech, and Signal Processing, Vol. ASSP-34 (1), 1986, pp. 21-27.

${ }^{13}$ Darlington, P., Wheeler, P.D., and Powell, G.A. "Adaptive Noise Reduction in Aircraft Communication Systems". Acoustics, Speech, and Signal Processing, IEEE International Conference on ICASSP, April, 1985.

${ }^{14}$ Piersol, A.G. "Use of Coherence and Phase Data between Two Receivers in Evaluation of Noise Environments". Journal of Sound and Vibration, Vol. 56 (2), 1978, pp. 215-228.

${ }^{15}$ Weiss, M.R., Aschkenasy, E., and Parsons, T.W. "Processing Speech Signals to Attenuate Interference", IEEE Symp. Speech Recognition, Apr. 1974.

${ }^{16}$ Humphreys Jr., W.M., Brooks, T.F., Hunter Jr., W.W., and Meadows, K.R. "Design and Use of Microphone Directional Arrays for Aeroacoustic Measurements". AIAA 1998-0471, $36^{\text {th }}$ Aerospace Sciences Meeting and Exhibit, Reno, NV, January, 1998.

${ }^{17}$ Brooks, T.F., and Humphreys Jr., W.M. "Effect of Directional Array Size on the Measurement of Airframe Noise Components". AIAA 1999-1958, $5^{\text {th }}$ AIAA/CEAS Aeroacoustics Conference, Bellevue, WA, May, 1999.

${ }^{18}$ Hutcheson, F.V. and Brooks, T.F., "Measurement of Trailing Edge Noise Using Directional Array and Coherent Output Power Methods", International Journal of Aeroacoustics. Vol. 1 (4), 2002, pp. 329-354.

${ }^{19}$ Brooks, T.F. and Humphreys, W.M., Jr., "Flap Edge Aeroacoustic Measurements and Predictions", Journal of Sound and Vibration, Vol. 261, 2003, pp. 31-74.

${ }^{20}$ Hutcheson, F.V. and Brooks, T.F., "Effects of Angle of Attack and Velocity on Trailing Edge Noise", AIAA Paper 2004 1031.

${ }^{21}$ Mosher, M. "Phased Arrays for Aeroacoustic Testing - Theoretical Development". AIAA 1996-1713, 2nd AIAA/CEAS Aeroacoustics Conference, State College, PA, May 1996.

${ }^{22}$ Brooks, T.F., and Humphreys, W.M. "A Deconvolution Approach for the Mapping of Acoustic Sources (DAMAS) Determined From Phased Microphone Arrays”. Journal of Sound and Vibration, Vol. 294, 2006, pp. 856-879.

${ }^{23}$ Bendat, J., and Piersol, A. Random Data, Analysis and Measurement Procedures. 2nd ed., Wiley, New York, 1986.

${ }^{24}$ Nelson, P.A., and Elliot, S.J. Active Control of Sound. Academic Press, London, 1992. 